\title{
1 Niche specificity, polygeny, and pleiotropy in herbivorous insects
}

$3 \quad{ }^{1}$ Nate B. Hardy and ${ }^{2}$ Matt Forister

5 Department of Entomology and Plant Pathology, Auburn University, Auburn, AL 36849

$6 \quad{ }^{2}$ Department of Biology, University of Nevada, Reno, NV 89557

\section{Abstract}

9 Why do herbivorous insects tend to be host specialists? Population genetic models predict

10 specialization when there is antagonistic pleiotropy at a gene for host-use performance. But

11 empirically, host-use performance is governed by many genetic regions, and antagonistic pleiotropy is

12 rare. Here, we use individual-based quantitative genetic simulations to investigate the role of pleiotropy

13 in the evolution of host-use specialization when host-use performance is polygenic. We find that if

14 host-preference is allowed to evolve without cost, parasite populations tend to evolve host-use

15 specialization even without pleiotropy; thus, it would seem that for a polygenic trait, the benefit of

16 maintaining adaptive combinations of conditionally-neutral alleles suffices to drive specialization. But

17 if there is a fecundity cost for host-preference, or if host patches are demographically volatile, host-use

18 generalists evolve, even with high levels of pleiotropy. In sum, if pleiotropy is much more pervasive

19 than has been observed in nature, our simulations show that it could play a role in driving the evolution

20 of polygenic specialization. But pleiotropy is not necessary, and even when it is extensive, selection can

21 favor generalist genotypes. 


\section{Introduction}

23 Most species of herbivorous insects use as hosts only a small fraction of the plant species they

24 encounter in their environment (Forister et al., 2015; Futuyma \& Moreno, 1988), despite the obvious

25 advantages of using many hosts and having a broader resource base (Hardy et al., 2020; Rainey et al.,

26 2000; Smith \& Hoekstra, 1980). How do we explain such host specificity? For the most part, theorists

27 have assumed that host specificity is a consequence of antagonistic pleiotropy (Egas et al., 2004;

28 Rueffler et al., 2004): an allele that confers high performance on one host confers low performance on another. Mathematical models show that if host-use performance is determined by one gene with antagonistic pleiotropy, and if populations can evolve host-use preferences, then the evolution of hostuse specialization is all but inevitable (Ravigné et al., 2009), barring pronounced temporal heterogeneity in the host environment that could make specialization risky. world. First, in herbivorous insects, most host-specific performance phenotypes, such as the ability to 


\section{Methods}

47 Simulations were performed with SliM 3 (Haller \& Messer, 2019). In this framework, models are specified in the Eidos programming language. Eidos codes are provided in S1-4. Before we describe our models in any detail, let us start with an overview. What we modeled was the mutational history of a population of diploid individuals that occurs in a simple environment consisting of two distinct resource patches. We imagined that these individuals were parasites, and that the resources patches were host species, called Host A and Host B. Individual host-use preference and performance

53 phenotypes evolved as a function of mutations at many genetic loci. We were primarily interested in

54 how changes in parasite genetic architecture - specifically, the ratio of pleiotropic to conditionally-

55 neutral mutations - affected subpopulation divergence across host species. By subpopulation

56 divergence we mean divergence at both neutral and host-use-affecting loci, with divergence at the latter sites reflecting the evolution of specialization.

Now let us look at the model details. As is typical for herbivorous insects, generations were discrete and non-overlapping. To keep things simple, individuals were hermaphroditic and mating was random. Selection was hard; the fitness, $\boldsymbol{W}$, of individuals was absolute (not relative to conspecifics).

61 Moreover, $\boldsymbol{W}$ determined the odds of survival rather than fecundity. (How this was calculated is described below.) The regulation of each host-associated subpopulation was density-dependent, with a nominal carrying capacity for each host patch of 500 individuals. But since fitness was absolute, it was possible for a patch to sustain populations above or below that threshold. In other words, population size was an emergent property of the model, reflecting the balance between individual survival rates and fecundity (with individual survival probabilities, described in greater detail below, scaled by the ratio of local patch carrying capacity to the current number of individuals in the local subpopulation). 
70 selection in which the survival probability of each individual was first determined by the match

71 between their genotype and environment, and then scaled by subpopulation density (Figure 1). At the

72 start of each generation, each surviving individual randomly selects as a mate one individual from their

73 subpopulation. The fecundity of the choosing individual is determined by a draw from a Poisson

74 distribution with a mean and variance $(\chi)$ of two. The selected number of crosses between a mating pair

75 (chooser and chosen) are then added to the subpopulation. Note that choosers could also be chosen as

76 mates by other individuals in their subpopulation (there is no limit to the number of matings an

77 individual could be involved in, but the chances of being involved in more than one or two matings is

78 small). Thus, individual fecundity could be much greater than that resulting from their own mate

79 selection.

After reproduction, each new individual selects a host patch to inhabit. Habitat choice is

81 determined in part by quantitative trait loci (QTLs). At the start of a simulation all individuals have no

82 preference, that is, an equal likelihood of choosing Host A or Host B. Then, as the simulation

83 progresses, host preference evolves as a function of the sum of mutational effects at preference-

84 determining loci. The realized host choice behavior of each individual is calculated as a weighted

85 average of their host-preference genotype and an inertial tendency to remain in their natal host patch.

86 The weights were $40 \%$ for host preference and $60 \%$ for host inertia. Here are some more technical

87 details. Host preferences phenotype values range from 0 to 1 . At the start of a simulation, each

88 individual has a preference genotype initialized at 0.5 . Then, each generation, preference genotypes are

89 updated as the sum of the initial genotype value (0.5) and the allele effects at preference loci. A

90 separate variable for preference inertia is set to 0 on Host A and 1 on Host B. As an example, if the

91 preference genotype value for an individual on Host B is 0.8 ( $0.5+$ the sum of preference loci mutation

92 effects), then overall preference becomes $0.4 * 0.8+0.6 * 1=0.92$. When this weighted average is less

93 than 0.5 , Host A is preferred; when greater than 0.5 the other host is preferred. Preference is then 
5

94 expressed by using the preference phenotype value as a cutoff for a draw from a random uniform

95 distribution. To continue the same example, if the random draw were 0.6 , the individual would choose

96 Host B, and if the random draw were 0.99 the individual would choose and migrate to Host A, although

97 such an outcome has a relatively low probability. There were no direct effects from host-preference

98 alleles on survival, thus, selection on those loci was indirect via the fitness effects of alleles that

99 determined performance on each host species (more on this later).

100 Now we turn to selection. On Host A, there is stabilizing selection on the quantitative

101 phenotype value of Trait 1 . One Host B, there is stabilizing selection on the phenotype value of Trait 2.

102 As a concrete example, we can think of these traits as plant-defensive-chemical-detoxification systems

103 - such as mixed-function oxidases and glutathione S-transferases (Enayati et al., 2005; Feyereisen,

104 1999) - with phenotype values corresponding to particular activity levels. Or the two traits could

105 represent two components of a parasite's immune response to pathogens with different optimal values

106 in different host-plant patches. Phenotypes are determined solely by genotypes, that is, by the additive

107 combination of QTLs. We assume no environmental effects on phenotypes, or dominance effects on

108 genotype values.

109 Each parasite individual has a diploid, $100 \mathrm{~kb}$, one-chromosome genome. This genome is

110 composed of ten $5 \mathrm{~kb}$ coding regions interspersed with ten $5 \mathrm{~kb}$ non-coding regions. Of course real

111 herbivorous insect genomes are much larger and have multiple chromosomes. Working with smaller

112 genomes is easier computationally, but increases linkage disequilibrium. To correct for that, we set the

113 recombination rate to 1e-4 events per base pair (bp) per generation. At that rate, we expect about ten

114 crossing-over events per genome per generation, which corresponds roughly to as much genetic

115 shuffling as would be typical for a real herbivorous insect species with a five-chromosome genome

116 (Wilfert et al., 2007). As alluded to previously, at the start of each simulation, all individuals have the

117 same ur-genome. The mutation rate is 1e-7 per bp per generation. Five types of mutations could occur: 
118 (1) neutral mutations in the non-coding regions, (2) pleiotropic mutations, that is, mutations at QTLs

119 that govern Traits 1 and 2, and thereby affected survival on both Host A and B, (3) conditionally neutral

120 mutations at QTLs that contribute to Trait 1 but not 2, (4) conditionally neutral mutations at QTLs that

121 contribute to Trait 2 but not 1, and (5) pleiotropic mutations that governed the host-preference trait.

122 Host-preference genes were mixed in with host-performance genes in the coding regions of the

123 genome.

When a mutation occurred at a QTL affecting either preference or performance, an effect was

125 drawn from a Gaussian distribution with a mean of zero and a variance $\boldsymbol{\sigma}$ which varied over

126 simulations taking the values $0.1,0.6$, and 1.2 . To be clear, the effect of an allele at a pleiotropic host-

127 performance QTL is the same in both host patches (i.e. symmetrical), determined by one draw from the

128 Gaussian distribution of effects. Whether the pleiotropy is negative or positive depends on the local

129 patch optimum along with an individual's genetic environment, that is, the sum of allele affects across

130 host-performance QTLs. Alternatively, we could have selected effects from a bivariate normal

131 distribution with a covariance more than zero, but less than one. This would have allowed for less

132 pronounced pleiotropies. We opted for maximally pronounced pleiotropies.

By the end of each simulation, the optimal performance trait value for each patch is displaced

134 by ten units from zero, the starting condition (see below). Thus, when the allele effect variance is 1.2,

135 the minimum number of averaged-sized mutations that it would take for a genotype value to match the

136 final patch optimum is about eight, whereas when the allele effect variance is 0.1 , the minimum is 100 .

137 But those are minimums. With each mutational effect drawn randomly from a Gaussian distribution,

138 and without selection, it could take many more mutations to sum to a genotype absolute value of ten,

139 and the disparity in the number of mutations needed when effect variances are small or large would be

140 more pronounced. At the end of our simulations, the mean number of segregating alleles was 77.02, 
7

141 with a standard deviation of 17.49 . The mean number that reached a frequency greater than $90 \%$ in each host patch was 35.18, with a standard deviation of 27.71 . host-patches diverged, (2) fecundity costs for strong host-use preference, and (3) the volatility of hostuse patches.

Model 1. In the first model variant, we explored the case of a gradual host patch divergence and no-cost host-preference. At the start, the optimal host-use phenotype value in each host patch was the same, zero, which was also the starting mean genotype value for the parasites population. Then, after every 100 generations, the optimal quantitative phenotype value on Host A increases by a value of 0.2 , 
meant that initial parasite populations were well-adapted to their host environment. And the gradual

divergence of host patch optima meant that host-use adaptation could proceed via incremental changes remained at zero for 1,000 generations, over which the strength of stabilizing selection was low, $\omega=$ 
189 accumulated genetic diversity that was cryptically pleiotropic (McGuigan \& Sgrò, 2009), that is, mutations that had no strong effect on fitness in the initial host environment, but that would have strong

191 and antagonistic effects in the novel host environment. We though that this model captured genetic

192 contingencies that could affect how a naive parasite population adapts to new hosts. We also suspected

193 that a population reservoir of cryptically pleiotropic alleles would increase the overall impact of

194 pleiotropy on host-use evolution.

Model 4. This model variant incorporated demographic stochasticity. Host-patch diverge was specialist subpopulation, whereas the same crash only shrinks a generalist population to 55\% of its pleiotropy got in the way.

We ran each simulation for 5,000 generations, with 100 replicates for each combination of

205 model parameters. For different perspectives on the genetic states of a metapopulation at the end of 206 each simulation, we logged these statistics: (1) $\boldsymbol{W}$ : mean population fitness; (2) $\boldsymbol{F}_{\text {ST: }}$ : the standard ratio 207 of between subpopulation neutral genetic variance to total neutral genetic variance; (3) the ratio of 208 fixed pleiotropic to fixed conditionally neutral mutations, (4) the ratio of the mean effect sizes of fixed 209 pleiotropic and conditionally neutral mutations, and (5) $\Delta W$, a specialization index that measures 210 subpopulation divergence, defined as the mean difference between individual fitness in the resident 211 patch and what the fitness of an individual would have been in the other host patch, scaled by the 
212 overall mean fitness of the metapopulation (with smaller values denoting more generalist populations

213 because the individual-level discrepancy in fitness between patches is less).

In summary, we began by looking at a situation in which we thought the evolution of

specialization was especially likely: where host-use phenotypes were rigid (without environmental changes to this situation that we suspected would be more favorable to the evolution of generalists, namely, where host-preference had a cost, and the carrying capacities of host patches were volatile.

\section{Results and Discussion}

223 For each of the four model variants described above, we give five plots, each showing how a metapopulation variable tends to change with increasing amounts of pleiotropy in the mutational mix, as well as how those changes depend on, $\boldsymbol{\sigma}$, the mean allele effect size. First we show the mean fitness, $W$, of individuals in each patch. In these plots, values near one denote near perfect matches between mean genotype values and local performance trait optima. To be clear, these plots just tell us how welladapted the metapopulation is; they tell us nothing about the degree to which adaptation entailed divergence of host-use phenotypes between subpopulations. Next we show mean $\boldsymbol{F}_{\boldsymbol{S T}}$ values. These can range between zero and one, with values closer to one denoting more pronounced neutral genetic

231 divergence between subpopulations. In the third plot, we show mean values of the specialization index, $232 \Delta \boldsymbol{W}$. As a reminder, this also ranges between zero and one, with higher values corresponding to more 233 pronounced divergence in host-use phenotypes between patches. In the fourth plot, we show the ratio 234 of fixed pleiotropic to fixed conditionally neutral mutations, and in the fifth plot, we show the ratio of 235 the mean effect sizes of the fixed pleiotropic and conditionally neutral mutations. 
Model 1. No-cost host-preference in gradually-diverging host environments (Figure 2)

238 For this model, divergence between host patches was gradual. At the end of 5,000 generations, when $\boldsymbol{\sigma}$

239 (a variance parameter that controls the distribution of mutations on performance alleles) was 0.6 or 1.2 adapted. But when $\boldsymbol{\sigma}$ was 0.1, it was $\sim 25 \%$ with lower with low-pleiotropy mutational mixes, and more effect size decreases, the average number of sites needed to produce an optimal genotype value increases; decreasing $\boldsymbol{\sigma}$ may tend to increase the effective polygeny of a trait. With more polygeny, the may become more challenging. Moreover, it would seem that this challenge becomes more significant with more pleiotropy use specificity) were near zero across the full range of mutational mixes; in other words, we ended up with poorly-adapted, genetically cohesive, generalist populations. When $\boldsymbol{\sigma}$ was 0.6 and pleiotropy was scant, $\boldsymbol{F}_{\boldsymbol{S T}}$ and $\Delta \boldsymbol{W}$ were low, that is we ended up with well-adapted generalist parasite populations. pleitropy in the mutational mix exceeded one. The picture was even more complicated when $\boldsymbol{\sigma}$ was 1.2 , up with well-adapted, relatively-specialized subpopulations. But when the amount of pleiotropy in the

258 plummeted; thus, it seems that in some conditions increasing pleiotropy can actually decrease 


\section{1 pleiotropic to fixed conditionally neutral mutations, and (2) the ratio of the mean effect sizes of fixed}

262 pleiotropic and conditionally-neutral mutations. We saw that, when pleiotropic mutations are relatively

263 rare, in comparison to conditionally neutral mutations, few pleiotropic mutations are fixed. Moreover,

264 those that are fixed tend to have much smaller effects, especially when $\boldsymbol{\sigma}$ is not overly small. Simply

265 put, selection purges large-effect pleiotropic mutations with extreme prejudice. It is only when

266 pleiotropies are introduced to a population at a high rate that they are likely to exert influence on the

267 adaptive fate of a population.

Models 2 and 3. Host-preference with a cost

270 In these models, strong host preference lowered individual fecundity. When the divergence of host-

271 patch optima was gradual (Model 2; Figure 3), we found that such a cost was sufficient to evolve high-

272 performance host-use generalists across a range of mutational mixes. As in the no-cost-preference

273 simulations, when $\boldsymbol{\sigma}$ was low, so too was mean fitness, and that became more pronounced with

274 increasing pleiotropy. Otherwise, fitness was near unity at the end of each simulation. The major

275 changes caused by the addition of a performance cost were that $\boldsymbol{F}_{\boldsymbol{S T}}$ and $\Delta \boldsymbol{W}$ were near zero, across the

276 range of allele effect sizes, until the ratio of pleiotropic to conditionally neutral mutations was greater

277 than 32-to-one - much higher than what has been observed for host-use traits in real plant-parasitic

278 insect populations. If host-preference is expensive, parasite populations tend to evolve no preference or 279 specialization.

What if host preference comes at a fecundity cost and the divergence in host-patch optima is

281 instantaneous, after a long period of overlap and stasis? To find out, we ran simulations in which host-

282 patches diverged all at once, and the strength of stabilizing selection increased dramatically after 1,000

283 generations. To reiterate, this lag could allow masked pleiotropic mutations to accumulate in a 
13

284 population, and thus increase the potential impact of pleiotropy. Sure enough, this did appear to

285

286

287

288

289

290

\section{No-cost host-preference in fluctuating host patches (Model 4; Figure 5)}

292

293

294

295

296

297

298

299

300

301 Without a doubt, we have explored the parameter space in a willy-nilly fashion. For example, we could

302

303

304

305

306

increase the range of mutational mix values over which pleiotropy increased parasite population

divergence and specialization. Interestingly, it also dramatically reduced the mean effect sizes of fixed

pleiotropic mutations. Thus, it seems that a naive population's mutational history can be a contingency

that forces specialization in a newly diverse host environment. Even so, selection purges large effect pleiotropic alleles.

We also looked to see what would happen if host-preference evolves without a fecundity cost, but the

carrying capacities of host patches were subject to stochastic crashes. As mentioned earlier, generalism should cut the fitness costs of such environmental volatility. What we found was similar to results from models in which host-use preference came with a fecundity cost. High-performance generalists evolved unless pleiotropic mutations were much more common than conditionally neutral mutations. The upshot is that we don't need direct costs for host preference to evolve polygenic generalism. The indirect cost on long-term fitness of specializing on a volatile resource is sufficient.

\section{Caveats and roads not taken}

have reported on a range of selection strengths and recombination rates, and we could have had a more fully-factorial set of simulations for the parameters that we did vary. Such a thorough exploration might have produced a thoroughly unreadable tome. Instead, our goal has been more modest: to further the idea that the importance of antagonistic pleiotropy in the evolution of specialization continues to play too central of a role in the thinking of most empirical and theoretical ecologists (Fry, 1996). To that 
end, we have attempted to point out a few neighborhoods in parameter space in which pleiotropy and or polygeny do or do not play roles in the evolution of host-use specificity. specified functions for mate choice, reproduction, habitat preference, and subpopulation-level densityuse phenotypic plasticity in nature.

\section{Conclusions}

320 If in herbivorous insect species the genetic architectures of traits affecting host-use performance were

321 simple, pleiotropy could explain the preponderance of host-use specificity. But they are not simple; the traits affecting host-use performance tend to be polygenic. Of course, the genetic architecture of host 
15

331 itself. The exception to that is if there are features of the genetic or external environment that add a cost

332 to specialization. Then we see the evolution of the generalists. Thus we suggest that future

333 experimental work on the evolution of specialization might not beat so loudly at the door of

334 antagonistic pleiotropy (or the absence thereof) but should look more broadly for costs to generalism

335 (Hardy et al., 2020).

336

337 Acknowledgments

338 Thanks to Zach Gompert, Josh Jahner, Gwendolyn Bird, and Noah Bevers for helpful comments on the

339 manuscript. This work was supporting in part by NSF award 1744552 to NBH and YYY to MF.

Author contributions

342

MF came had the premise. NB and MF together developed the models and wrote the manuscript. NB ran the simulations.

\section{References}

Egan, S. P., Ragland, G. J., Assour, L., Powell, T. H. Q., Hood, G. R., Emrich, S., Nosil, P., \& Feder, J. L. (2015). Experimental evidence of genome-wide impact of ecological selection during early stages of speciation-with-gene-flow. Ecology Letters, 18(8), 817-825. https://doi.org/10.1111/ele.12460

Egas, M., Dieckmann, U., \& Sabelis, M. W. (2004). Evolution restricts the coexistence of specialists and generalists: The role of trade-off structure. American Naturalist, 163(4), 518-531. https://doi.org/10.1086/382599

Enayati, A. A., Ranson, H., \& Hemingway, J. (2005). Insect glutathione transferases and insecticide resistance. In Insect Molecular Biology (Vol. 14, Issue 1, pp. 3-8). John Wiley \& Sons, Ltd. https://doi.org/10.1111/j.1365-2583.2004.00529.x 
Feyereisen, R. (1999). INSECT P450 ENZYMES. Annual Review of Entomology, 44(1), 507-533. https://doi.org/10.1146/annurev.ento.44.1.507

Forister, M. L., Novotny, V., Panorska, A. K., Baje, L., Basset, Y., Butterill, P. T., Cizek, L., Coley, P. D., Dem, F., Diniz, I. R., Drozd, P., Fox, M., Glassmire, A. E., Hazen, R., Hrcek, J., Jahner, J. P., Kaman, O., Kozubowski, T. J., Kursar, T. A., ... Dyer, L. A. (2015). The global distribution of diet breadth in insect herbivores. Proceedings of the National Academy of Sciences of the United States of America, 112(2), 442-447. https://doi.org/10.1073/pnas.1423042112

Fry, J. D. (1996). The Evolution of Host Specialization: Are Trade-Offs Overrated? The American Naturalist, 148, S84-S107. https://doi.org/10.1086/285904

Futuyma, D. J., \& Moreno, G. (1988). The Evolution of Ecological Specialization. Annual Review of Ecology and Systematics, 19, 207-233.

Gompert, Z., Jahner, J. P., Scholl, C. F., Wilson, J. S., Lucas, L. K., Soria-Carrasco, V., Fordyce, J. A., Nice, C. C., Buerkle, C. A., \& Forister, M. L. (2015). The evolution of novel host use is unlikely to be constrained by trade-offs or a lack of genetic variation. Molecular Ecology, 24(11), 27772793. https://doi.org/10.1111/mec.13199

Haller, B. C., \& Messer, P. W. (2019). SLiM 3: Forward Genetic Simulations Beyond the Wright-Fisher Model. Molecular Biology and Evolution, 36(3), 632-637.

https://doi.org/10.1093/molbev/msy228

Hardy, N. B., Kaczvinsky, C., Bird, G., \& Normark, B. B. (2020). What We Don’t Know About DietBreadth Evolution in Herbivorous Insects. Annual Review of Ecology, Evolution, and Systematics, 51(1). https://doi.org/10.1146/annurev-ecolsys-011720-023322

Jaenike, J. (1990). Host Specialization in Phytophagous Insects. Annual Review of Ecology and Systematics, 21, 243-273.

McGuigan, K., \& Sgrò, C. M. (2009). Evolutionary consequences of cryptic genetic variation. Trends in Ecology and Evolution, 24(6), 305-311. https://doi.org/10.1016/j.tree.2009.02.001

Rainey, P. B., Buckling, A., Kassen, R., \& Travisano, M. (2000). The emergence and maintenance of diversity: insights from experimental bacterial populations. Trends in Ecology \& Evolution, 15(6), 243-247. https://doi.org/10.1016/S0169-5347(00)01871-1

Ravigné, V., Dieckmann, U., \& Olivieri, I. (2009). Live where you thrive: joint evolution of habitat choice and local adaptation facilitates specialization and promotes diversity. American Naturalist, 174(4). https://doi.org/10.1086/605369

Rueffler, C., Van Dooren, T. J. M., \& Metz, J. A. J. (2004). Adaptive walks on changing landscapes: Levins’ approach extended. Theoretical Population Biology, 65(2), 165-178. https://doi.org/10.1016/j.tpb.2003.10.001 
17

Smith, J. M., \& Hoekstra, R. (1980). Polymorphism in a varied environment: how robust are the models? Genetics Research, 35(01), 45. https://doi.org/10.1017/S0016672300013926

Wilfert, L., Gadau, J., \& Schmid-Hempel, P. (2007). Variation in genomic recombination rates among animal taxa and the case of social insects. Heredity, 98(4), 189-197. https://doi.org/10.1038/sj.hdy.6800950

\section{Figures}

Figure 1. Simulated parasite life cycle. Each generation consists of a round of random mate choice followed by reproduction, in which parent genomes are mutated and crossed, and then offspring are added to the occupied host patch. After reproducing, parents die. The environment consists of two host plant species. Offspring chose to stay on their natal host species, or migrate to the other host species. This decision is a function of an inertial tendency to stay where they are, and their host preference genotypes, which are determined by the sum of allele effects at many QTLs. Selection determines survival probabilities; survival is more likely for individuals that have quantitative host-use genotypes that more closely match the optimum value on their host plant species.

\section{Figure 2. Model 1. No-cost host-preference in gradually-diverging host environments. Coral-}

colored lines are for mean allele effect sizes, $\boldsymbol{\sigma}$, of 1.2, green lines are for $\boldsymbol{\sigma}$ of 0.6 , and blue lines are for $\boldsymbol{\sigma}$ of 0.1. (A) Mean fitness, $\boldsymbol{W}$, of individuals across parasite metapopulation. Values near one denote near perfect matches between mean genotype values and local performance trait optima. (B) Mean $\boldsymbol{F}_{\boldsymbol{S T}}$ values. Values closer to one denoting more pronounced neutral genetic divergence between subpopulations. (C) Mean values of the specialization index, $\Delta W$, values closer to one denote more pronounced divergence in host-use phenotypes between patches. (D) Ratio of fixed plieotropic to fixed conditionally neutral mutations. (E) Ratio of the mean effect sizes of the fixed pleiotropic and conditionally neutral mutations. 
Figure 3. Model 2. Expensive host-preference in gradually-diverging host environments. Coral-

421 colored lines are for mean allele effect sizes, $\boldsymbol{\sigma}$, of 1.2, green lines are for $\boldsymbol{\sigma}$ of 0.6 , and blue lines are for $\boldsymbol{\sigma}$ of 0.1. (A) Mean fitness, $\boldsymbol{W}$, of individuals across parasite metapopulation. Values near one denote near perfect matches between mean genotype values and local performance trait optima. (B) Mean $\boldsymbol{F}_{\boldsymbol{S} \boldsymbol{T}}$ values. Values closer to one denoting more pronounced neutral genetic divergence between conditionally neutral mutations.

431 Coral-colored lines are for mean allele effect sizes, $\boldsymbol{\sigma}$, of 1.2, green lines are for $\boldsymbol{\sigma}$ of 0.6 , and blue lines

432 are for $\boldsymbol{\sigma}$ of 0.1 . (A) Mean fitness, $\boldsymbol{W}$, of individuals across parasite metapopulation. Values near one conditionally neutral mutations. 
19

443 near perfect matches between mean genotype values and local performance trait optima. (B) Mean $\boldsymbol{F}_{\boldsymbol{S T}}$

444 values. Values closer to one denoting more pronounced neutral genetic divergence between

445 subpopulations. (C) Mean values of the specialization index, $\Delta W$, values closer to one denote more

446 pronounced divergence in host-use phenotypes between patches. (D) Ratio of fixed plieotropic to fixed

447 conditionally neutral mutations. (E) Ratio of the mean effect sizes of the fixed pleiotropic and

448 conditionally neutral mutations. 
A

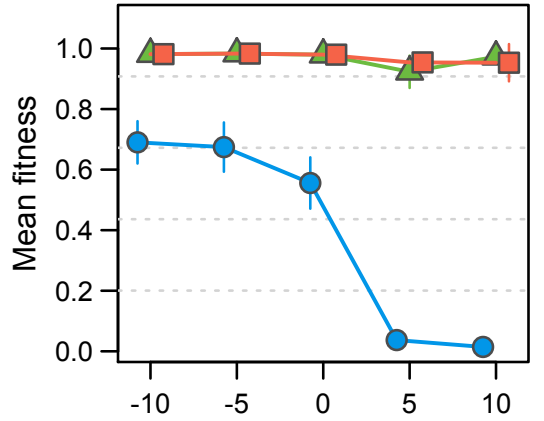

B

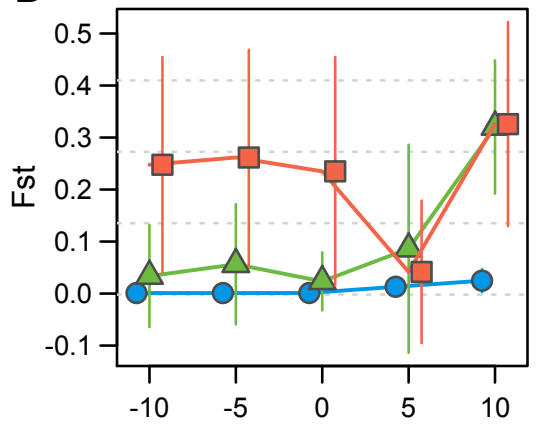

C

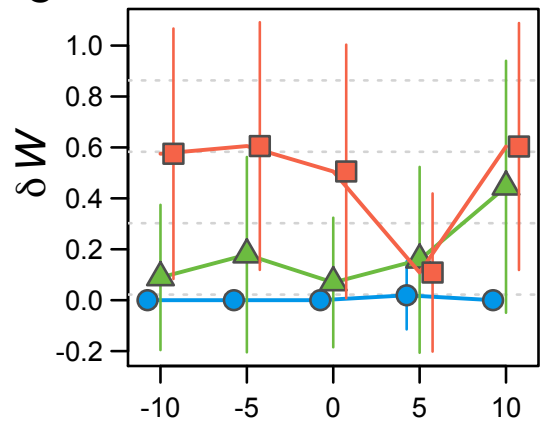

D
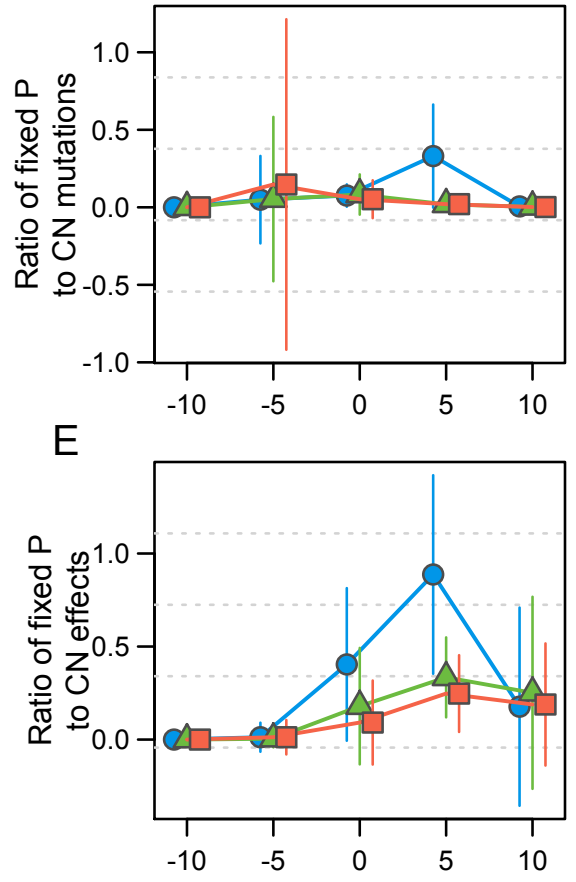

Ratio of Pleiotropic to $\mathrm{CN}$ mutations

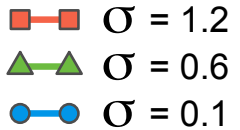

Ratio of Pleiotropic to $\mathrm{CN}$ mutations 
A
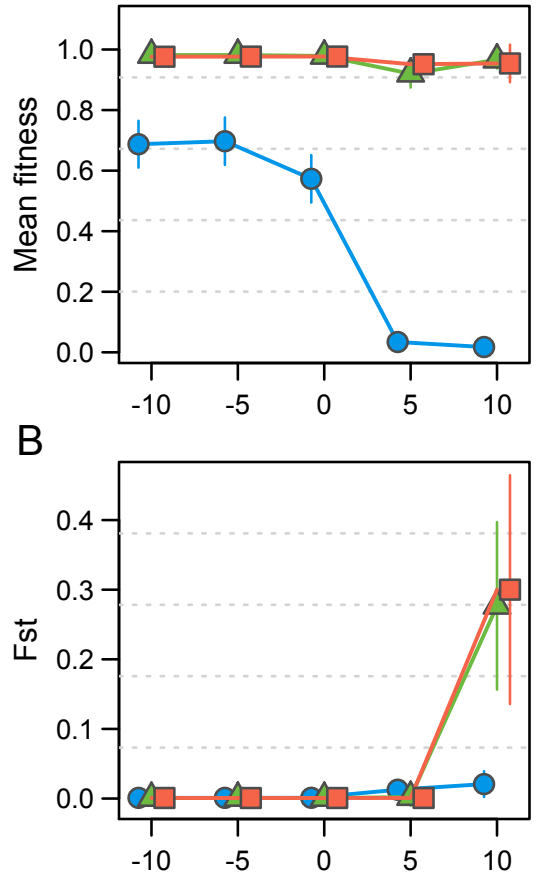

C

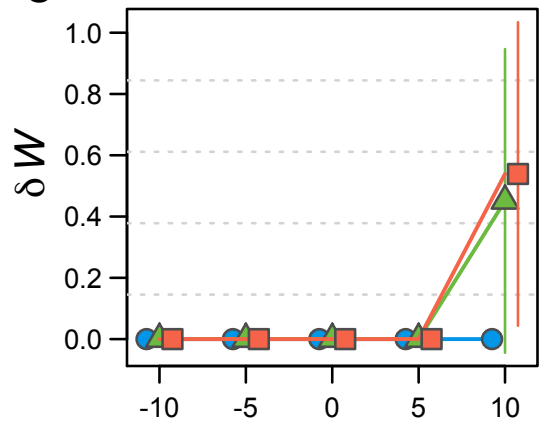

D

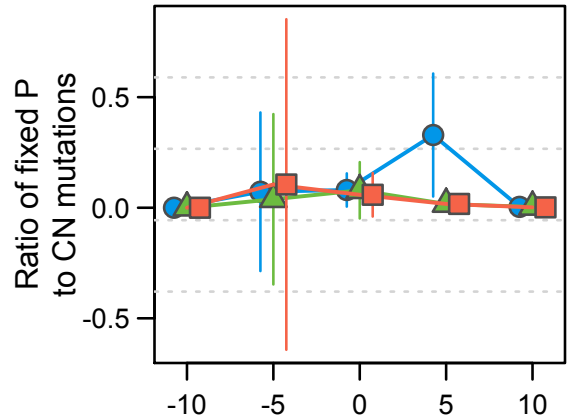

E

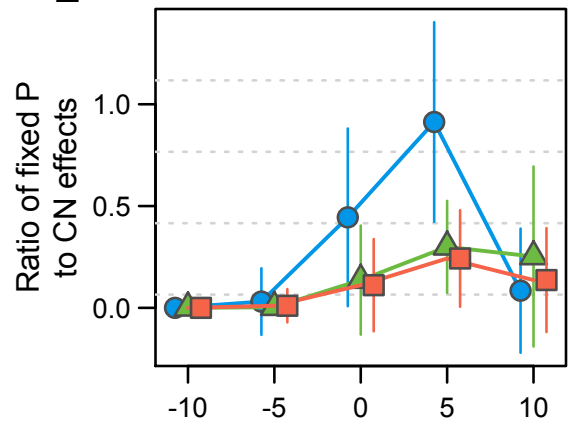

Ratio of Pleiotropic to $\mathrm{CN}$ mutations

$$
\begin{aligned}
& \square \square \sigma=1.2 \\
& \triangle \triangle \sigma=0.6 \\
& \square \sigma=0.1
\end{aligned}
$$

Ratio of Pleiotropic to $\mathrm{CN}$ mutations 
A

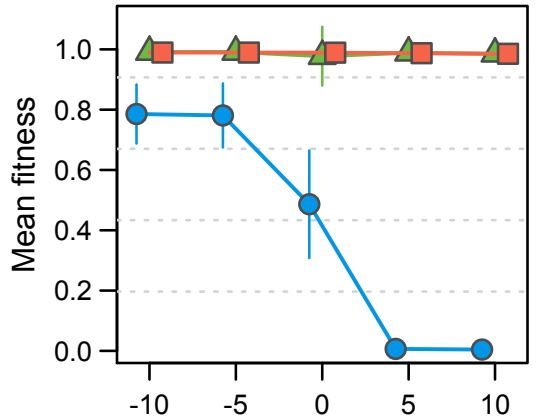

B

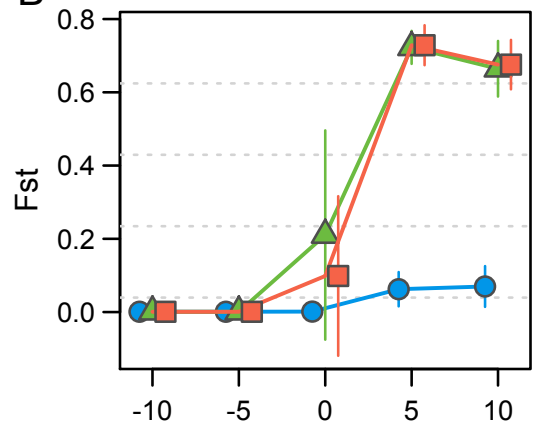

C

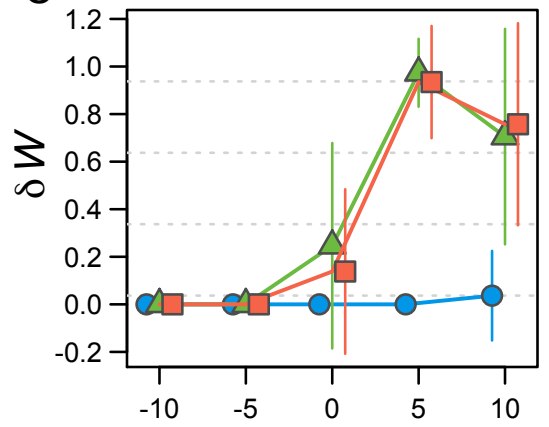

D

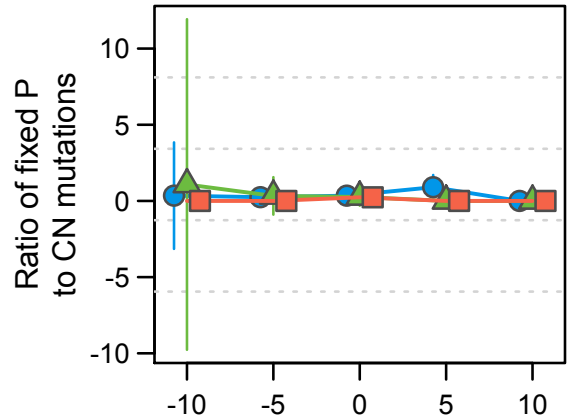

E

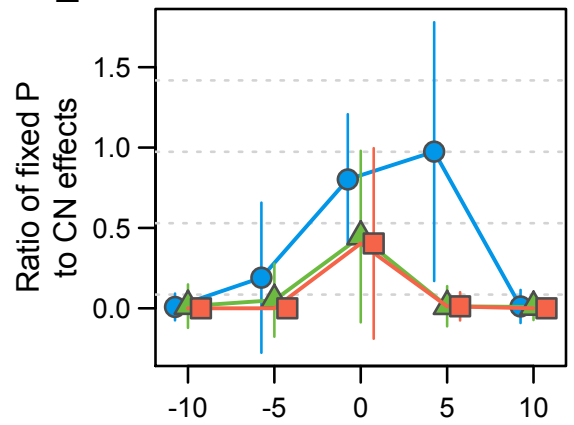

Ratio of Pleiotropic to $\mathrm{CN}$ mutations $\square \square \sigma=1.2$
$\triangle \triangle \sigma=0.6$
$\square \sigma=0.1$

Ratio of Pleiotropic to $\mathrm{CN}$ mutations 
A

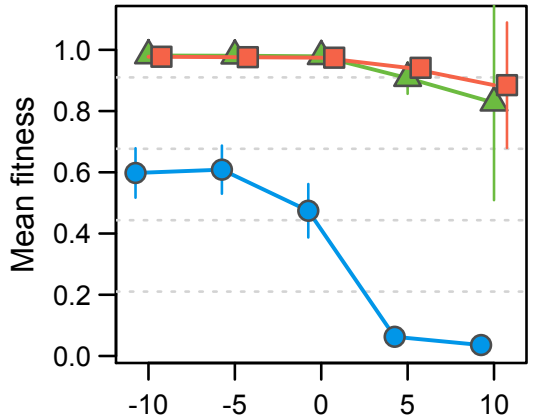

B

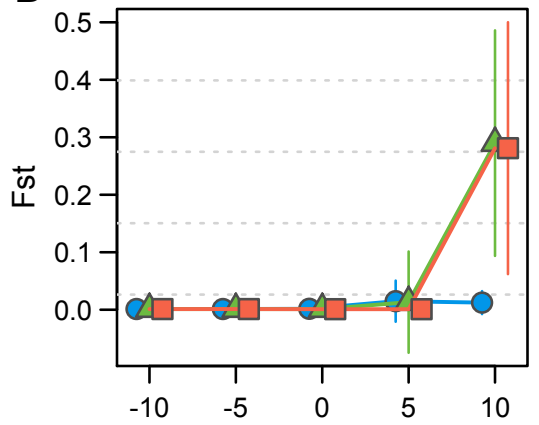

C

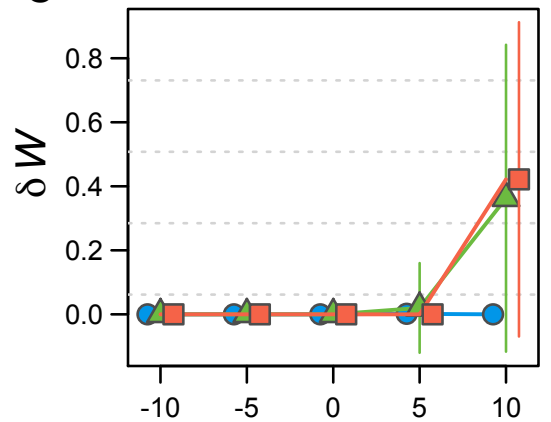

D
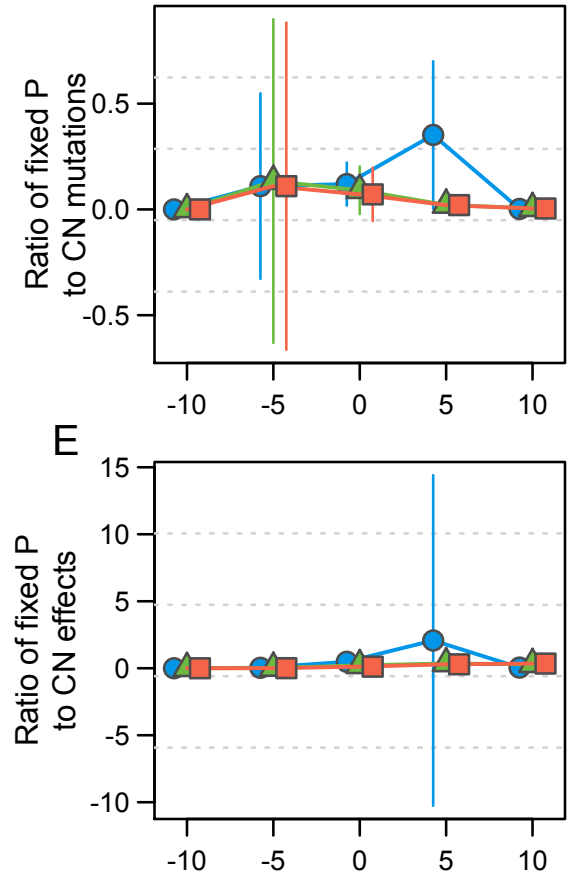

Ratio of Pleiotropic to $\mathrm{CN}$ mutations

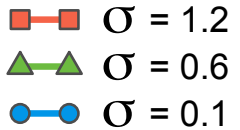

Ratio of Pleiotropic to $\mathrm{CN}$ mutations 